\title{
Novel Sequence Variants in the NPC1 Gene in Egyptian Patients with Niemann-Pick Type C
}

\author{
Mona L. Essawi ${ }^{1}$, Asmaa F. Abdel-Aleem ${ }^{1 \star}$, Mohamed A. Badawy ${ }^{2}$, Maha S. Zaki ${ }^{3}$, Magda F. Mohamed ${ }^{4,5}$, Heba A. Hassan $^{1}$, \\ Ekram M. Fateen ${ }^{6}$ \\ ${ }^{1}$ Department of Medical Molecular Genetics, Human Genetics and Genome Research Division, National Research Centre, \\ Cairo, Egypt; ${ }^{2}$ Department of Organic Chemistry, Faculty of Science, Cairo University, Giza, Egypt; ${ }^{3}$ Department of Clinical \\ Genetics, Human Genetics and Genome Research Division, National Research Centre, Cairo, Egypt; ${ }^{4}$ Department of Chemistry \\ (Biochemistry Branch), Faculty of Science, Cairo University, Giza, Egypt; ${ }^{5}$ Department of Chemistry, Faculty of Science and \\ Arts, Khulais, University of Jeddah, Jeddah, Saudi Arabia; ${ }^{6}$ Department of Biochemical Genetics, Human Genetics and Genome \\ Research Division, National Research Centre, Cairo, Egypt
}

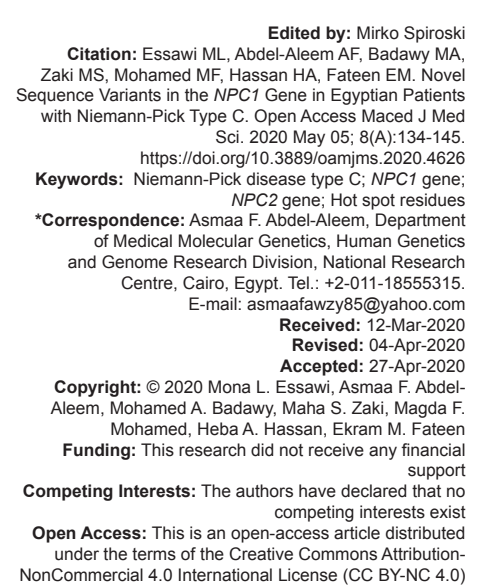

\section{Introduction}

Niemann-Pick disease type C (NPC, OMIM\# 257220) is a progressive pan-ethnic autosomal recessive lysosomal lipid storage disorder, with an estimated incidence of 1 in 100,000 live birthsworldwide [1]. It is characterized by impaired intracellular lipid trafficking leadingto the aggregation of unesterified cholesterol and sphingolipids in different body organs such as liver, spleen, and brain [2]. The disease has an extremely heterogeneous clinical presentation, includes visceral abnormalities, neurological, and psychiatric manifestations that appear alone or in combination with various ages of onset [3]. Splenomegaly with or without hepatomegalyand prolonged neonatal jaundice are common visceral manifestations. The classic neurological symptoms mainly include "cerebellar ataxia, dysarthria, dysphagia, cataplexy, and progressive dementia" in addition to vertical supranuclear gaze palsy
(VSGP) which represents a major disease characteristic feature [4].

NPC is caused mainly by a biallelic mutation in either of NPC1 (95\% of cases) or NPC2 (4\% of cases) gene [5]. NPC1 is located on chromosome 18q11-12, contains 25 exons, and encodes a 1278 amino acid glycoprotein with 13 transmembrane domains [6]. Up till now, more than 400 various NPC1 mutations have been described in NPC patients, including insertions, deletions, duplications, nonsense, and missense mutations [7]. Although these mutations are scattered throughout the gene, the cysteine-rich luminal loop (amino acid residues: 857-1015) was reported as a hot spot regionencountering around one-third of the revealed mutations. While, in other literature, the putative sterol sensing domain was reported as a second hot spot region, including around $20 \%$ of revealed mutations [8]. In addition, the amino acid residues(1038-1253) in the c-terminal carboxyl end of protein were also previously mentionedas a hot spotfor pathogenic variants. Some 
NPC1 mutations showed recurrent distribution globally or among different populations. p.lls1061Thr is the most common reported mutation and widely distributed in Western Europe. p.Pro1007Ala is reported as the second most recurrent mutationin different populations, especially in Germany. p.Arg1186His is widely distributed inthe Czech, while Gly992Trp is individualized to Nova Scotian patients [9].

Disease-specific drugs such as miglustat can slow down neurological disease progression [10]. New therapeutic approaches such as gene therapy showed promising results in vitro and in vivo and it is supposed to be applied in the clinical stage in the near future [11]. In our study, we investigated certain clinical, biochemical, and molecular features in 15 NPC Egyptian patientsto contribute a better disease characterization in Egypt. We aimed at the characterizationof certain NPC1 hotspot regions (residues: 855-1098 and 1038-1253), especially they also encounter the most common NPC1 mutations worldwide, hopefully, to contribute in disease diagnosis confirmation and proper genetic counseling among NPC Egyptian patients.

\section{Patients and Methods}

The current caseseries study was conducted on 15 NPC Egyptian patients with an age range between 6 months and 10 years(mean \pm SD, 3.2 \pm 3 years)andsome selected parents as well as 20 healthy controls with matched age and sex during the period from September 2017 to November 2019.

The patients were referred to the clinical genetics and biochemical genetics departments at the National Research Center (NRC), Cairo, Egypt. All cases were diagnosed as NPC according to their clinical presentation and biochemical studies. They were also subjected to history taking include: Age of patients, sex, age of disease onset, and consanguinity. Blood samples were obtained from patients after having written informed approval from their parents or their legal guardians. The study protocol was approved by the Medical Research Ethics Committee at NRC.

\section{Clinical examinations}

All included patients were clinically diagnosed, according to Millat et al. [12]. Abdominal ultrasonography, magnetic resonance imaging (MRI) of the brain, and electroencephalography were performed for all patients.

\section{Biochemical studies}

Peripheral leukocytic $\beta$-glucocerebrosidase and peripheral leukocytic acid sphingomyelinase enzymes were measured to exclude similar metabolic diseases such as Gaucher and Niemann-Pick Type A and Type B diseases [13], [14]. Evaluation of chitotriosidase biomarker as a recommended screening tool for NPC was done [15].

\section{Molecular studies}

DNA extraction

DNA was extracted from peripheral blood lymphocytes from 15 NPC patients, selected parents, and 20 healthy controls by salting out technique [16]. The DNA concentration was measured at a $260 / 280 \mathrm{~nm}$ absorbance ratio by NanoDrop 2000c Spectrophotometer (Thermo Fisher).

\section{Polymerase chain reaction (PCR) amplification of NPC1 exons (17-25)}

PCR was performed in a total volume of $30 \mu$ containing 100-200 ng genomic DNA, 30 pmol of each primer,230 $\mu \mathrm{M}$ of dATP, dCTP, dTTP, and dGTP, 2.5 $\mu \mathrm{M} \mathrm{Mgcl2,} 1 \mathrm{X}$ Taq buffer and $2.5 \mathrm{U}$ Taq polymerase. PCR was performed for nineexons of NPC1 gene. The primers sequencewas previously described byTarugi et al. [17], Table S1. Amplification of the nine exons was performed with an initial denaturation step at $95^{\circ} \mathrm{C}$ for $5 \mathrm{~min}$ followed by 35 cycles (each comprising $94^{\circ} \mathrm{Cfor} 40 \mathrm{~s}, 65^{\circ} \mathrm{Cfor}$ $40 \mathrm{~s}$, and $72^{\circ} \mathrm{Cfor} 40 \mathrm{~s}$ ) and a final extension step at $72^{\circ} \mathrm{Cfor} 10 \mathrm{~min}$. The reaction was carried out in BioRad thermal cycler. Each PCR product was verifiedby electrophoresis on a $1.5 \%(\mathrm{w} / \mathrm{v})$ agarose gel and visualization by UV light.

\section{Purification of the PCR product}

PCR products were purified using EXO/SAP PCR Purification Kit(Thermo Fisher Scientific Baltics, UAB, Lithuania);2 $\mu \mathrm{l}$ EXO/SAP cocktail (4 U Exo I and $0.4 \mathrm{U}$ SAP) were added to $6 \mu \mathrm{PCR}$ product. The mix was incubated at $37^{\circ} \mathrm{Cfor} 60 \mathrm{~min}$, then at $80^{\circ} \mathrm{Cfor}$ $20 \mathrm{~min}$.

\section{(17-25)}

\section{Direct DNA sequencing of NPC exons}

PCR amplified fragments were sequenced in both directions using primers and a BigDye Terminator Cycle Sequencing Kit v1.1 (Applied Biosystems, Warrington, UK), samples were run on an $A B I$ PRISM Genetic Analyzer 3500 (Applied Biosystems). Sequences were compared using Basic Local Alignment Search Tool [18].

All variants were described according to nomenclature recommendation by the human genome variations society [19]. Sequence analysis of NPC1was 
analyzed regarding the reference sequence of(GenBank accession no. NPC1, NM_000271.4).

\section{In silico analysis}

All annotated variants were examined in several databases, including ClinVar [20], HGMD [7], and gnomAD databases [21].

Functional prediction analysis, including PolyPhen2 [22], SIFT [23], and Mutation Taster [24], was used to evaluate the functional effect of novel nonsynonymous amino acid substitutions. NPC1mutation tolerance was further analyzed usingthe MetaDome webserver [25].

Prediction of protein structural effect after novel nonsynonymous variations was performed by Project HOPEto predict structural variation between native protein and the variant model [26]. Protein sequence and structure were retrieved from UniProt "Universal Protein Resource" [27]. The UniProt ID number of the aligned human sequences of NPC1: "UniProt: 015118."

\section{Results}

\section{Clinical data}

This study included 15 Egyptian patients belongingto15 unrelated families. Consanguinity was certain in 12 families $(80 \%)$. They were 9 males $(60 \%)$ and 6 females $(40 \%)$ and their age ranged from 6 months to 10 years(mean \pm SD, $3.2 \pm 3$ years). The onset of the disease varies from early infantile to juvenile form. Accordingly, our patients were classified into early infantile (<2 years) in 7 patients $(46.7 \%$ ), late infantile (2-5 years) in 4 patients (26.7\%), and juvenile (5-16 years) in 1 patient $(6.7 \%)$. Hepatosplenomegaly was the most common disease systemic feature in $(60 \%)$ of cases, while splenomegaly restricted to $33.3 \%$ of them. Neurological manifestations were obvious in 12 patients $(80 \%)$, classified as early infantile-onset $(58.3 \%)$, late infantile-onset (33.3\%), and juvenile-onset (8.3\%). Dysphagia, cataplexy, hypotonia, delayed developmental milestones, speech retardation, loss of previously acquired skills, ataxia, epilepsy, and vertical supranuclear gaze palsy VSGP were themain observable neurological manifestations. Brain MRI was performed in all patients, around $(46.7 \%)$ of casesshowed cerebral and cortical atrophy, whilemild cortical atrophic changeswere observed in $(33.3 \%)$, on the other hand around $(20 \%)$ showed normal MRI. Detailed clinical data are illustrated in Table 1.

\section{Biochemical studies}

Assessment of peripheral leukocytic $\beta$-glucocerebrosidase and peripheral leukocytic acid sphingomyelinase enzymes showed normal values in all patients, while a remarkable elevation in chitotriosidase activitywas observed in all patients(Table S2).

\section{Molecular studies}

Molecular analysis of 9 exons of NPC1 (exons 17-25) confirmed sixdifferent mutations. Two homozygous novel missense variants in the amino acid residues (1038-1253) were detected and they have already been submitted to ClinVar under accessions SCV001197997 and SCV001197998. The novel mutations were p.Ser1169Arg in the cytoplasmic loop $\mathrm{L}$ and $\mathrm{p}$.Ser1197Phe in the $12^{\text {th }} \mathrm{TM}$ domain (Figure 1). Molecular analysis of parents showed heterozygous status for the novel variants, and the 20 healthy individuals showed wild type sequences. Four previously reported mutations were detected in the cysteine-rich luminal loop classified as, one nonsense homozygous mutationp. Arg958*, one heterozygous missense mutation p.Gly910Ser, additionally, two homozygous frameshift mutations p.Ala927Glyfs*38, p.Cys1011* (Figure S1). Positions of novel revealed variants can be visualized in NPC1 protein topology produced by Protter software (Figure S2) [28].

\section{In silico results}

All revealed variants were not identified in the overall populations as referenced inthe gnomADdatabase expect p.Gly910Serand p.Arg958*which showed minor allele frequency (0.000007953)and (0.000003982), respectively. Both mutations were also reported as pathogenic variants in the ClinVar database. The novel sequence variations were evaluated through the alignment of amino acid sequences from eight different organisms and alterations are located within conserved residues suggesting an effect on proteins functions or structures (Figure S3). Pathogenicity of novel missense variations was confirmed using different web-based tools (PolyPhen2, SIFT, and Mutation Taster). According to the guidelines by the American College of Medical Genetics and Genomics, both of p.Ser1169Arg and p.Ser1197phe were found to be likely pathogenic variants. MetaDome web server showed that two novel variants are located in regions intolerant for missense variation. According to project HOPE, novel missense variantsp. Ser1169Arg and p.Ser1197Phe were predicted to make harmful effects on proteins binding and folding processes causing subsequently protein malfunction (Figure 2). In silico analysis, results are all summarized in (Table 2). 


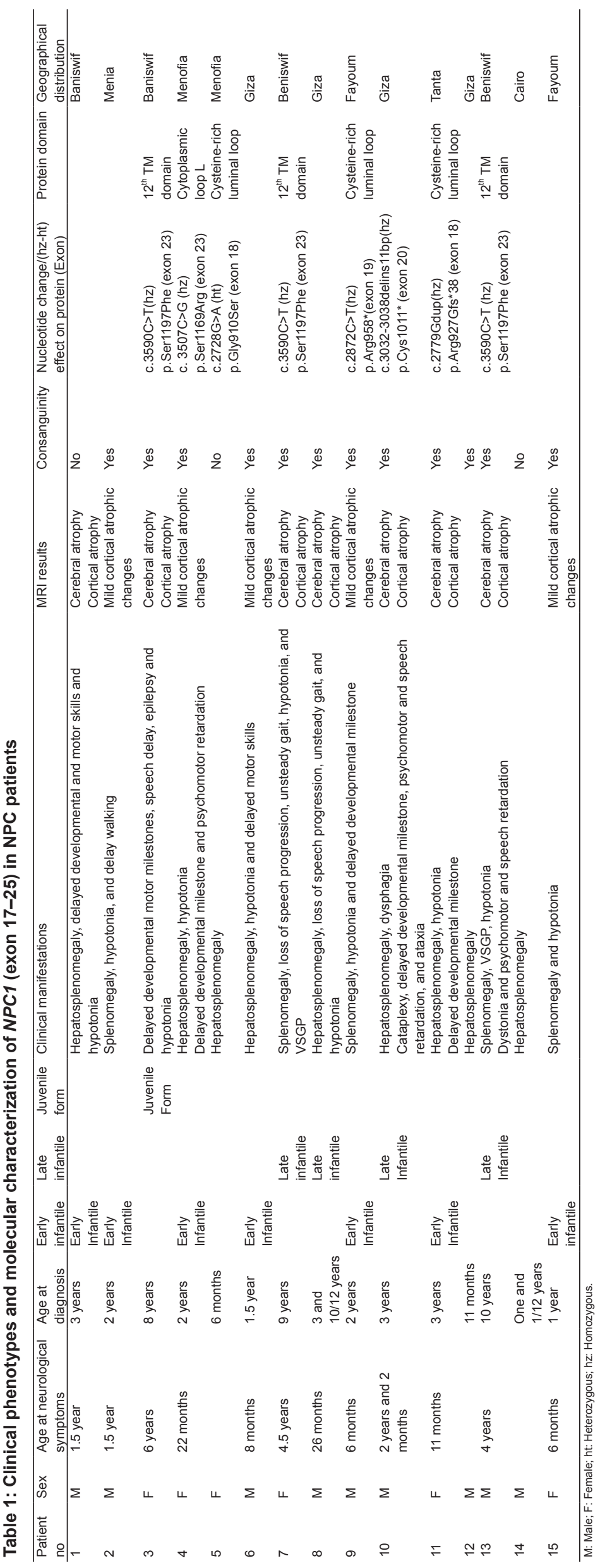




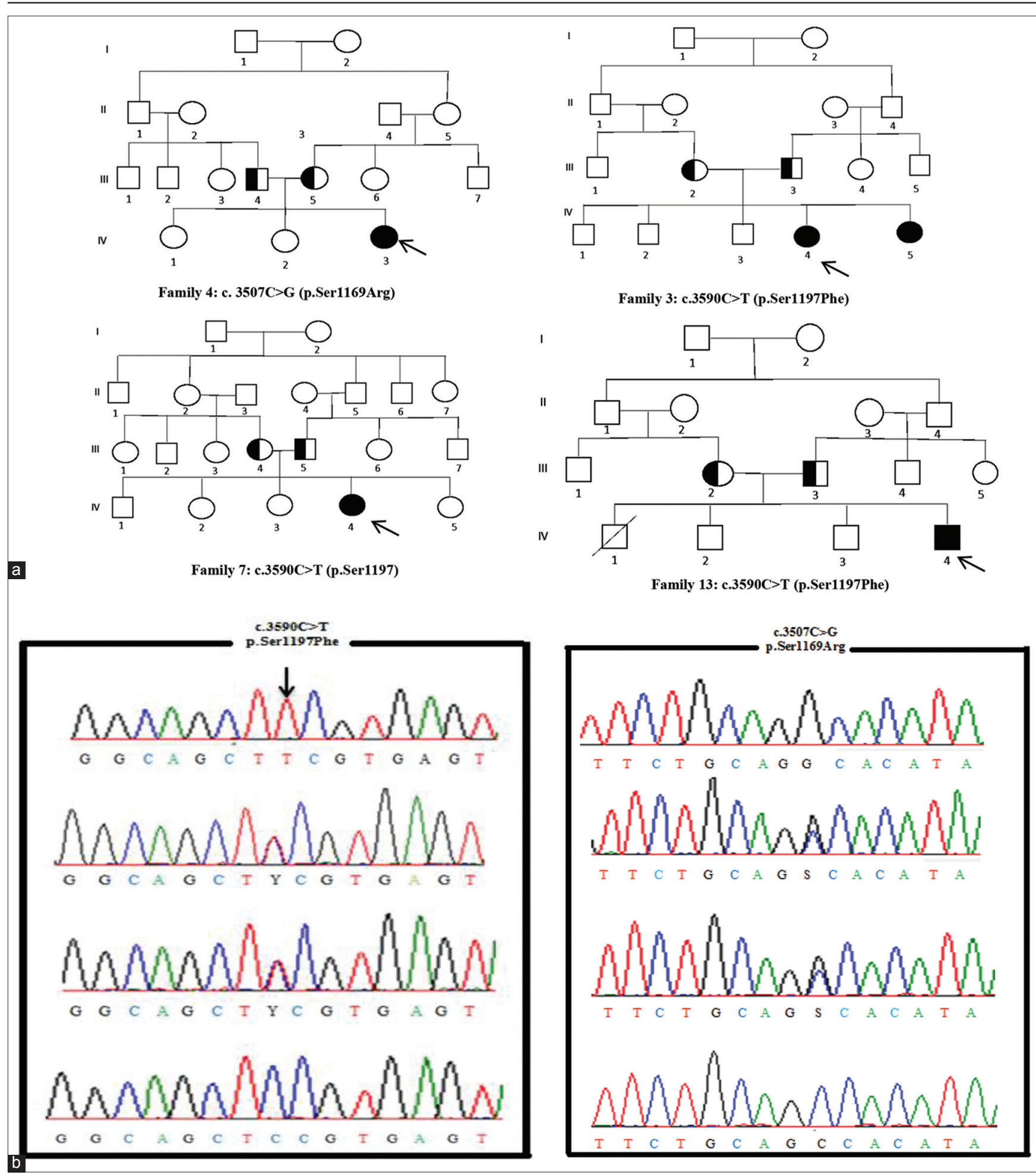

Figure 1: Pedigrees and chromatograms of four families 4, 3, 7, 13. (a) Squares indicate males; circles indicate females; the black symbols indicate affected individuals; the half-black symbols indicate carriers, arrows indicate the molecularly studied probands. (b) Novel variants of NPC1 in 4 probands. The upper chromatogram in each frame represents the variant sequence, the lower one depicts the reference sequence, and the two middle sequences represent the parent's heterozygous sequences

\section{Discussion}

NPCis an autosomal recessive lysosomal storage diseasemainly caused by homozygous or compounds heterozygous mutations in NPC1 $195 \%$ of cases) [1]. Early disease diagnosis is very crucial for proper initiation of treatment and inhibition of disease progression [3]. In the current study, 15 Egyptian patients clinically and biochemically diagnosed as NPC. As previously mentioned, NPC exhibits broad clinical heterogeneity and wide presentation [29], which is illustrated herein. Ourpatients showed a remarkable phenotypic variability and their age ranged from 
Table 2: In silico analysis of novel revealed variants

\begin{tabular}{|c|c|c|c|c|c|c|c|c|}
\hline $\begin{array}{l}\text { cDNA variant } \\
\text { (NM_000271.4) }\end{array}$ & $\begin{array}{l}\text { Mutation effect on protein } \\
\text { (NP 000262.1) }\end{array}$ & GnomAD & PolyPhen-2 & SIFT & Mutation taster & MetaDome tolerance & ClinVar & HOPE \\
\hline c. $3590 \mathrm{C}>\mathrm{T}$ & p.Ser1197Phe & 0 & Probably damaging & Deleterious & Pathogenic & Slightly intolerant & No entry & Protein malfunction \\
\hline c. $3507 \mathrm{C}>\mathrm{G}$ & p.Ser1169Arg & 0 & Probably damaging & Deleterious & Pathogenic & Intolerant & No entry & Protein malfunction \\
\hline
\end{tabular}

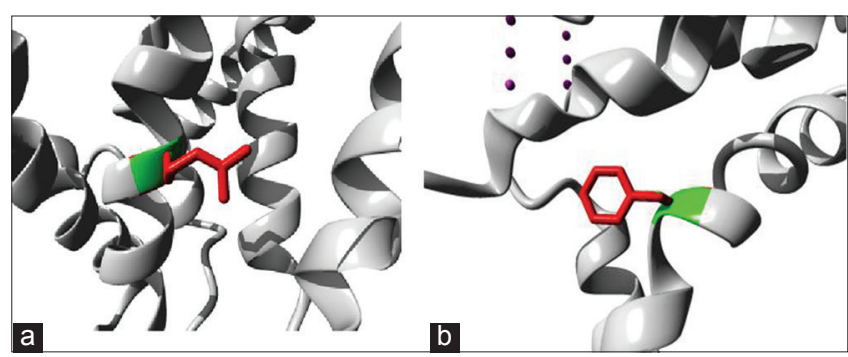

Figure 2: Structural variation between native protein and the variant models according to hope project, (a) zoom in variantp. Ser1169Arg, the protein is colored gray, the side chains of the wild type (serine) and the mutant (Arginine) residue are colored green and red, respectively, (b) zoom in variant p.Ser1197Phe, the protein is colored gray; the side chains of the wild type (serine) and the mutant (phenylalanine) residue are colored green and red, respectively

6 months to 10 years old. Around $80 \%$ ofcases were diagnosed after the presence of neurologicalmanifestations, while the diagnosis was only established according to systemic signs in other cases $(20 \%)$, systemic manifestations such as hepatosplenomegaly or splenomegaly were obvious in the majority of cases $(93.3 \%)$ this is consistent with previous literature where systemic manifestations areusually present preceding to neurological onsetwith the exception of few percentages of patients(approximately,15\%) [30]. The early infantileonset was the most common form in our study; it represents(46.5\%) of cases followed by the late infantile-onset $(26.7 \%)$ while the juvenile onset was only present in $(6.7 \%)$ of cases. This agrees with the previous studies that reported the early infantile form as the most frequent disease form in Southern Europe ( $>20 \%$ of cases) and the Middle East [30]. However, a previous Egyptian study reported the neonatal form as a predominating form, followed by an early infantile onset of disease [31]. Remarkably, although severe neonatal cholestatic jaundice is more common in classic NPC, this feature was absent in the current study. Herein, the majority of pediatric patients' manifestations ( $\leq 4$ years) such as splenomegaly, hepatosplenomegaly, hypotonia, delayed neuro-psychomotor, ataxia, and cataplexy are in agreement with the common previously reported NPC signs in pediatric patients [1]. Clinical manifestations observed in included patients ( $>4$ years) such as cognitive decline, epilepsy, and VSGP were more commonly described in younger NPC patients [30]. Denoting the biochemical analysis, high chitotriosidase levels were detected in all included patients, which is a useful initial screening tool for disease diagnosis. Although other previous studies showed an elevated level of chitotriosidase in NPC patients, other assessments are required for proper diagnosis, as it is not a specific NPC diagnostic marker [32]. Consequently, disease diagnosis must be finally confirmed by molecular analysis, which isalso considered as the only major tool for prenatal diagnosis, family studies, and carrier detection. Up till now, more than 400 diseasecausing NPC1 mutationshave been reported [33]. Although they are widely scattered over the geneother studies considered some regions such as residues(855-1098) in the cysteine-rich luminal loop and residues(1038-1253) which shares $34.7 \%$ with PTC1, as hot spot regions for NPC disease mutations[34]. In this study, molecular analysis of nine exons (17-25) of NPC1 encountering the previously suggested hot spot residues(855-1098) and (10381253) revealed six different mutations (15 mutant alleles) in eightunrelated patients. Regarding the high consanguinity rate in our population, homozygous mutations were predominated. Patients showed different geographical distributions, they originatefrom different governorates all over Egypt. Around 23\% $(7 / 30)$ of the 30 studied alleles showed variations in the cysteine-rich luminal loop (residues 855-1098), while $26.7 \%(8 / 30)$ of them showed alterations at the amino acid residues(1038-1253). Distribution of mutations in the two studied amino acid residues (855-1098 and 1038-1253)was consistent with the previously described [34], [35]; moreover, a recent Egyptian study showed that around $37 \%$ of detected mutant alleles were in the currently studied hotspot regions [31], confirming that they can also be considered as hot spot regions in NPC Egyptian patients. Although the missense mutations p.lle1061Thr, p.Arg1186His, p.Pro1007Ala, and Gly992Trp were previously reported as the most common NPC mutations [9], none of them were detected in the present study;however, p.lle1061Thr was reported in the heterozygous state coupled with p.Ser627Arg in an Egyptian patient with the late infantile onset of disease [31]. Among severely affected patients, we identified one nonsense homozygous mutation, p.Arg958* inpatient9 led to protein truncation in exon 19 within the cysteine-rich luminal loop between TM8 and 9. The patient showed early infantile onset of disease, splenomegaly was the main systemic feature, while neurological manifestations such as hypotonia and delayed developmental milestone started to appear at the age of 6 months, the same mutation was also reported in a previous Egyptian study in two unrelated patients with neonatal and infantile onset of disease [31]. However, it was observed in the compound heterozygous state in a case with adult-onset of disease in a study applied in Western Europe and the USA [36]. This illustrates the severity of the mutation in the disease progression, as all the reported cases carryingthe homozygous p.Arg958* mutation was of early disease onset. However, the case 
previously mentioned reported by Bauer et al., [36], the neurological manifestations appeared in the adult stage due to the less severe missense mutation p.Asp874Val. Two frameshift mutations (c.3032 3038delins10bp p.Cys $1011^{*}$ ) and (c.2779dupG p.Ala927Glyfs*38) were separately observed in the homozygous form in patients 10 and 11, respectively, both mutations led to losing of essential domains of NPC1 protein and subsequent protein function impairment. Patient 10 showed a late infantile onset of disease and was suffering from hepatosplenomegaly in the early stage of life, while after 2 years of age, progressive neurological manifestations began to appear such as, dysphagia, cataplexy, delayed developmental milestone, psychomotor, speech retardation, and ataxia. Patient 11 was presented with hepatosplenomegaly, hypotonia, and delayed developmental milestone by the age of 2 years, with an early infantile onset of disease. The latter two frameshift mutations were not reported before in any populations rather than Egyptians. Two previously reported that Egyptian patients were described with the early infantile onset of disease [31]. Among cases with a late infantile-onset, we identified the novel missense variation p.Ser1169Arg in patient 4 , her parents were both heterozygous carriers and showed normal phenotypes. The patient was suffering from hepatosplenomegaly, hypotonia, delayed developmental milestones, and psychomotor retardation that appeared after 2 years of age. The mutation p.Ser1169Arg is located in the cytoplasmic loop $\mathrm{L}$ between the $11^{\text {th }}$ and $12^{\text {th }}$ transmembrane domains. Functional effect prediction programs confirmed the possible pathogenesis of this novel variant. Moreover, it is found to be located within conserved regions when multiple alignment analysis was applied in eightdifferent species. Within the same region, p.Cys 1168Tyr mutation was previously detected in a patient with the late infantile onset of disease, where immunoblotting of the mutated NPC1 protein showed a diminished amount of the protein [35]. This confirms the essential role of this region within the cytoplasmic loop $L$ for the protein activity process. On the other hand, these predictions were confirmed by project HOPE, which illustrated the difference in size, charge, and hydrophobicity value between the wild residue serine and mutant arginine. The exchange of the buried neutral serine residue with larger positively charged arginine could abolish the core structure of the domain and affect protein binding. In addition, loss of hydrophobic interaction between the protein core and membrane lipids may be developed due to the less hydrophobic mutant residue arginine, which affects protein function. Another novel missense variant p.Ser1197Phe was observed in a homozygous state in three different patients (3, 7, and 13). Although, they were not relatives; the three patients were from the same village. Sequencing of parents showed the heterozygous status of the variant. Patients 7 and 13had a late-infantile onset of disease and showed nearly similar manifestations. Splenomegaly was the main systemic feature, while neurological manifestations began after 4 years of age and included loss of speech progression, unsteady gait, and hypotonia in addition to VSGP. Concerning patient3, no systemic signs could be observed; she showed the juvenile form of the disease. Although three latter patients share the same variantp. Ser1197Phe, patient 3 showed different disease onset and clinical manifestations which highlight the extreme phenotypic heterogeneity of the disease. Despite considerable advances in biochemical andgenetic understanding of the disease, the cause of extensivephenotypic variability in NPC remains unclear [29]. The detected variant Ser1197phe is located within the $12^{\text {th }}$ transmembrane domain in a highly conserved region was confirmed by multiple alignment analysis. Functional effect prediction programs such asSIFT, PolyPhen2, andMutationTasterpredicted splice site alteration and possible pathogenicity after this novel variant. According to project HOPE, phenylalanine residue is larger and more hydrophobic than serine residue which may affect the protein contact with the lipid-membrane. In addition, in wild protein, the buried serine residue forms a hydrogen bond with glutamine at position 707, while in the mutant phenylalanine residue; loss of hydrogen bonds in the protein core is acquired dueto the difference in size and hydrophobicity of phenylalanine, leading to disruption of the protein correct folding.

Finally, mutation p.Gly910Ser was observed in the heterozygous state in patient 5 (6 months of age) who only suffered from hepatosplenomegaly while neurological manifestations havenot yet manifested on clinical evaluation, the same mutation was also reported in the heterozygous state coupled with p.Cys516Tyr mutation in a newborn infant with acute liver failure to parents of mixed Armenian and Turkish descent [37]. It was also observed in a patient with a juvenile form of the disease in the heterozygous state with p.Pro474Leu missense mutation in a previous Italian study [17]. Here, we could not detect the other heterozygous variant suggesting that patient may have another variant either in a more proximal portion of the NPC1or in NPC2.

\section{Conclusion}

Our study as one of the limited molecular studies on NPC1 gene of NPC Egyptian patients was able to investigate certain clinical, biochemical, and molecular characteristics in patients. It provides valuable information to enhance the recognition of some variations associated with NPC disease in Egyptians. The novel NPC1 variants p.Ser1169Arg and p.Ser1197Phe were mostly correlated with the late-infantile onset of the disease. Distribution of detected mutations revealed 
that the two studied amino acid residues (855-1098 and 1038-1253) could be considered as potentialhot spot regions in NPC1 Egyptian patients. Although NPC was variably distributed all over Egypt, a recurrent mutation within the same village illustrates the harmful impact of consanguineous marriage which would extend further from the unit of the family. Finally, the use of in silico bioinformatics tools for analysis of the pathogenicity of novel variants isa helpful, time-saving, and relatively accurate test. However, functional analysis of protein would be of great importance, especially in studies examining the disease pathogenesis and pathway.

\section{Ethical Statement}

This research was approved by the scientific ethics committee at the NRC.

\section{Informed Consent}

The patients' parents or their legal guardiansand controlswere consulted and agreed to participate in the study. Written informed consent was obtained from them.

\section{Acknowledgments}

The authors are grateful to the patients and their families and volunteers who kindly accepted to participate in our study.

\section{References}

1. Polese-Bonatto $\mathrm{M}$, Bock $\mathrm{H}$, Farias AC, Mergener R, Matte MC Gil MS, et al. Niemann-pick disease Type C: Mutation spectrum and novel sequence variations in the human NPC1 gene. Mol Neurobiol. 2019;56(9):6426-35.https://doi.org/10.1007/ s12035-019-1528-z

\section{PMid:30820861}

2. Vanier MT, Gissen P, Bauer P, Coll MJ, Burlina A, Hendriksz CJ, et al. Diagnostic tests for niemann-pick disease Type $C$ (NP-C): A critical review. Mol Genet Metab. 2016;118(4):244-54. https://doi.org/10.1016/j.ymgme.2016.06.004 PMid:27339554

3. Vanier MT. Niemann-pick disease Type C. OrphanetJRare Dis. 2010;5(1):16.https://doi.org/10.1186/1750-1172-5-16 PMid:20525256

4. Geberhiwot T, Moro A, Dardis A, Ramaswami U, Sirrs S,
Marfa MP, et al. Consensus clinical management guidelines for Niemann-pick disease Type C. Orphanet J Rare Dis. 2018;13(1):50.

\section{PMid:29625568}

5. Dardis A, Zampieri S, Gellera C, Carrozzo R, Cattarossi S, Peruzzo P, et al. Molecular genetics of Niemann-pick Type C disease in Italy: An update on 105 patients and description of 18 NPC1 novel variants. J Clin Med. 2020;9(3):679.

PMid:32138288

6. Bounford KM, Gissen P. Genetic and laboratory diagnostic approach in Niemann pick disease Type C. J Neurol. 2014;261(2):569-75.https://doi.org/10.1007/s00415-014-7386-8 PMid:25145893

7. Stenson PD, Ball EV, Mort M, Phillips AD, Shiel JA, Thomas NS, et al. Human gene mutation database (HGMD): 2003 update. HumMutat. 2003;21(6):577-81.https://doi.org/10.1002/ humu.10212

PMid:12754702

8. Yang CC, Su YN, Chiou PC, Fietz MJ, Yu CL, Hwu WL, et al. Six novel NPC1 mutations in Chinese patients with Niemann-pick disease Type C. J Neurol Neurosurg Psychiatry. 2005;76(4):592-5.https://doi.org/10.1136/jnnp.2004.046045 PMid:15774455

9. Bountouvi E, Papadopoulou A, Vanier MT, Nyktari G, Kanellakis S, Michelakakis $\mathrm{H}$, et al. Novel NPC1 mutations with different segregation in two related Greek patients with Niemannpick Type $C$ disease: Molecular study in the extended pedigree and clinical correlations. BMC Med Genet. 2017;18(1):51. https://doi.org/10.1186/s12881-017-0409-4 PMid:28472934

10. Pineda M, Walterfang M, Patterson MC. Miglustat in Niemannpick disease Type $\mathrm{C}$ patients: A review. Orphanet J Rare Dis. 2018;13(1):140.https://doi.org/10.1186/s13023-018-0844-0 PMid:30111334

11. Hughes $M$, Smith $D$, Morris L, Tordo J, Palomar-Martin N Henckaerts E, et al. Development of gene therapy for Niemannpick Type $C$ disease.In: Annual Conference of the British Society for Geneand Cell Therapy. London, England: University College London; 2016.

12. Millat G, Baïlo N, Molinero S, Rodriguez C, Chikh K, Vanier MT Niemann-pick $C$ disease: Use of denaturing high performance liquid chromatography for the detection of NPC1 and NPC2 genetic variations and impact on management of patients and families. Mol Genet Metab. 2005;86(1-2):220-32.https://doi. org/10.1016/j.ymgme.2005.07.007

PMid:16126423

13. DanielsLB, GlewRH, RadinNS, VunnamRR.Arevisedfluorometric assay for Gaucher's disease using conduritol- $\beta$-epoxide with liver as the source of $\beta$-glucosidase. Clin Chim Acta. 1980;106(2):15563.https://doi.org/10.1016/0009-8981(80)90168-0

14. Van Diggelen O, Voznyi YV, Keulemans JL, Schoonderwoerd K Ledvinova J, Mengel $\mathrm{E}$, et al. A new fluorimetric enzyme assay for the diagnosis of Niemann-pick $A / B$, with specificity of natural sphingomyelinase substrate. $\mathrm{J}$ Inherit Metab Dis. 2005;28(5):733-41.https://doi.org/10.1007/s10545-005-0105-y PMid:16151905

15. Hollak C, van Weely S, van Oers MH, Aerts JM. Marked elevation of plasma chitotriosidase activity. A novel hallmark of gaucher disease. J Clin Invest. 1994;93(3):1288-92.https://doi. org/10.1172/jci117084

PMid:8132768

16. Miller S, Dykes DD, Polesky H. A simple salting out procedure for extracting DNA from human nucleated cells. Nucleic Acids Res. 1988;16(3):1215.https://doi.org/10.1093/nar/16.3.1215 PMid:3344216 
17. Tarugi P, Ballarini G, Bembi B, Battisti C, Palmeri S, Panzani F, et al. Niemann-pick Type C disease mutations of NPC1 gene and evidence of abnormal expression of some mutant alleles in fibroblasts. J Lipid Res. 2002;43(11):1908-19.https://doi. org/10.1194/jlr.m200203-jlr200

\section{PMid: 12401890}

18. Pruitt KD, Katz KS, Sicotte H, Maglott DR. Introducing Re Seq and Locus Link: Curated human genome resources at the NCBI. Trends Genet. 2000;16(1):44-7.https://doi.org/10.1016/ s0168-9525(99)01882-x

19. denDunnen JT, Dalgleish R, Maglott DR, Hart RK, Greenblatt MS, McGowan-Jordan J, et al. HGVS recommendations for the description of sequence variants: 2016 update. Hum Mutat. 2016;37(6):564-9.https://doi.org/10.1002/humu.22981 PMid:26931183

20. Landrum MJ, Lee JM, Benson M, Brown G, Chao C, Chitipiralla S, et al. ClinVar: Public archive of interpretations of clinically relevant variants. Nucleic Acids Res. 2015;44(D1):D862-8. https://doi.org/10.1093/nar/gkv1222 PMid:26582918

21. Karczewski K, Francioli L. The Genome Aggregation Database (gnomAD). Boston, MA: MacArthur Lab; 2017.

22. Adzhubeil A, Schmidt S, Peshkin L, Ramensky VE, Gerasimova A, Bork P, et al. A method and server for predicting damaging missense mutations. Nat Methods. 2010;7(4):248. PMid:20354512

23. Sim NL, Kumar P, Hu J, Henikoff S, Schneider G, Ng PC. SIFT web server: Predicting effects of amino acid substitutions on proteins. Nucleic Acids Res. 2012;40(W1):W452-7.https://doi. org/10.1093/nar/gks539

PMid:22689647

24. Schwarz JM, Cooper DN, Schuelke M, Seelow D. Mutation Taster2: Mutation prediction for the deep-sequencing age. Nat Methods. 2014;11(4):361. PMid:24681721

25. Wiel L, Baakman C, Gilissen D, Veltman JA, Vriend G, Gilissen C. Meta Dome: Pathogenicity analysis of genetic variants through aggregation of homologous human protein domains. Hum Mutat. 2019;40(8):1030-8.https://doi.org/10.1101/509935 PMid:31116477

26. Venselaar H, TeBeek TA, Kuipers RK, Hekkelman ML, Vriend G. Protein structure analysis of mutations causing inheritable diseases. An e-Science approach with life scientist friendly interfaces. BMC Bioinformatics. 2010;11(1):548.https://doi. org/10.1186/1471-2105-11-548

PMid:21059217

27. Consortium U. UniProt: A hub for protein information. Nucleic Acids Res. 2014;43(D1):D204-12.https://doi.org/10.1093/nar/ gku989 PMid:25348405

28. Omasits $\mathrm{U}$, Ahrens $\mathrm{CH}$, Müller $\mathrm{S}$, Wollscheid B. Protter: Interactive protein feature visualization and integration with experimental proteomic data. Bioinformatics. 2013;30(6):884-6. https://doi.org/10.1093/bioinformatics/btt607

PMid:24162465

29. Benussi A, Alberici A, Premi E, Bertasi V, Cotelli MS, Turla M, et al. Phenotypic heterogeneity of Niemann-pick disease Type $C$ in monozygotic twins. J Neurol. 2015;262(3):642-7.

PMid:25536905

30. Vanier MT. Niemann-pick C disease: History, current research topics, biological and molecular diagnosis. Arch Pediatr. 2010;17Suppl 2:S41-4.

PMid:20620894

31. Mahmoud IG, Elmonem MA, Elkhateeb NM, Elnaggar W, Sobhi A, Girgis MY, et al. Clinical, biomarker and genetic spectrum of Niemann-pick Type $\mathrm{C}$ in Egypt: The detection of nine novel NPC1 mutations. Clin Genet. 2019;95(4):537.https:// doi.org/10.1111/cge.13492/v1/review2 PMid:30633340

32. De Castro-Oros I, Irún P, Cebolla JJ, Rodriguez-Sureda V, Mallén M, Pueyo MJ, et al. Assessment of plasma chitotriosidase activity, CCL18/PARC concentration and NP-C suspicion index in the diagnosis of Niemann-pick disease Type C: A prospective observational study. J Transl Med. 2017;15(1):43.https://doi. org/10.1186/s12967-017-1146-3

PMid:28222799

33. Shammas H, Kuech EM, Rizk S, Das AM, Naim HY. Different Niemann-pick C1 genotypes generate protein phenotypes that vary in their intracellular processing, trafficking and localization. Sci Rep. 2019;9(1):5292.https://doi.org/10.1038/ s41598-019-41707-y

34. Park WD, O'Brien JF, Lundquist PA, Kraft DL, Vockley CW, Karnes PS, et al. Identification of 58 novel mutations in Niemann-pick disease Type C: Correlation with biochemical phenotype and importance of PTC1-like domains in NPC1. Hum Mutat. 2003;22(4):313-25.https://doi.org/10.1002/humu.10255 PMid: 12955717

35. Millat G, Marçais C, Tomasetto C, Chikh K, Fensom AH Harzer K, et al. Niemann-pick C1 disease: Correlations between NPC1 mutations, levels of NPC1 protein, and phenotypes emphasize the functional significance of the putative sterolsensing domain and of the cysteine-rich luminal loop. Am J Hum Genet. 2001;68(6):1373-85.https://doi.org/10.1086/320606 PMid:11333381

36. Bauer $P$, Balding DJ, Klünemann $\mathrm{HH}$, Linden DE, Ory DS, Pineda $M$, et al. Genetic screening for Niemann-pick disease Type $C$ in adults with neurological and psychiatric symptoms: Findings from the ZOOM study. Hum Mol Genet. 2013;22(21):4349-56.https://doi.org/10.1093/hmg/ddt284 PMid:23773996

37. Rohanizadegan M, Abdo SM, O'Donnell-Luria A, Mihalek I, P, Sanders $\mathrm{M}$, et al. Utility of rapid whole-exome sequencing in the diagnosis of Niemann-pick disease Type $\mathrm{C}$ presenting with fetal hydrops and acute liver failure. Cold Spring Harb Mol Case Stud. 2017;3(6):a002147.https://doi.org/10.1101/mcs.a002147 PMid:28802248 


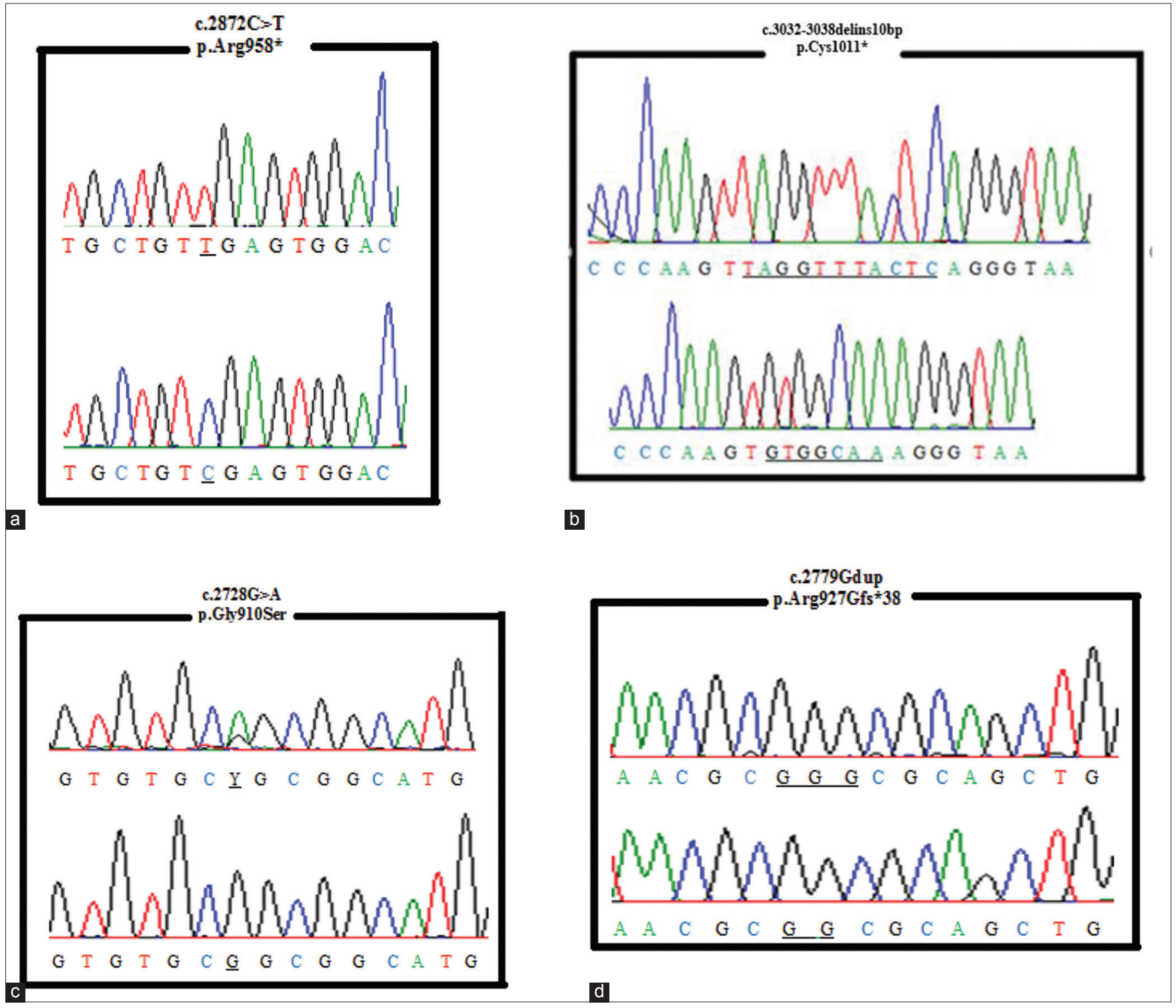

Figure S1: The revealed reported mutations of NPC1 (a) represents the homozygous nonsense mutation p.Arg958* detected in patient 9; (b) shows the homozygous frameshift mutation p.Cys1011* detected in patient 10; (c) shows the heterozygous missense mutation p.Gly910Ser revealed in patient 5; (d) represents the homozygous frameshift mutation p.Arg927Gfs 38 observed in patient 11 . The upper chromatogram in each frame represents the variant sequence; the lower one shows the reference sequence 


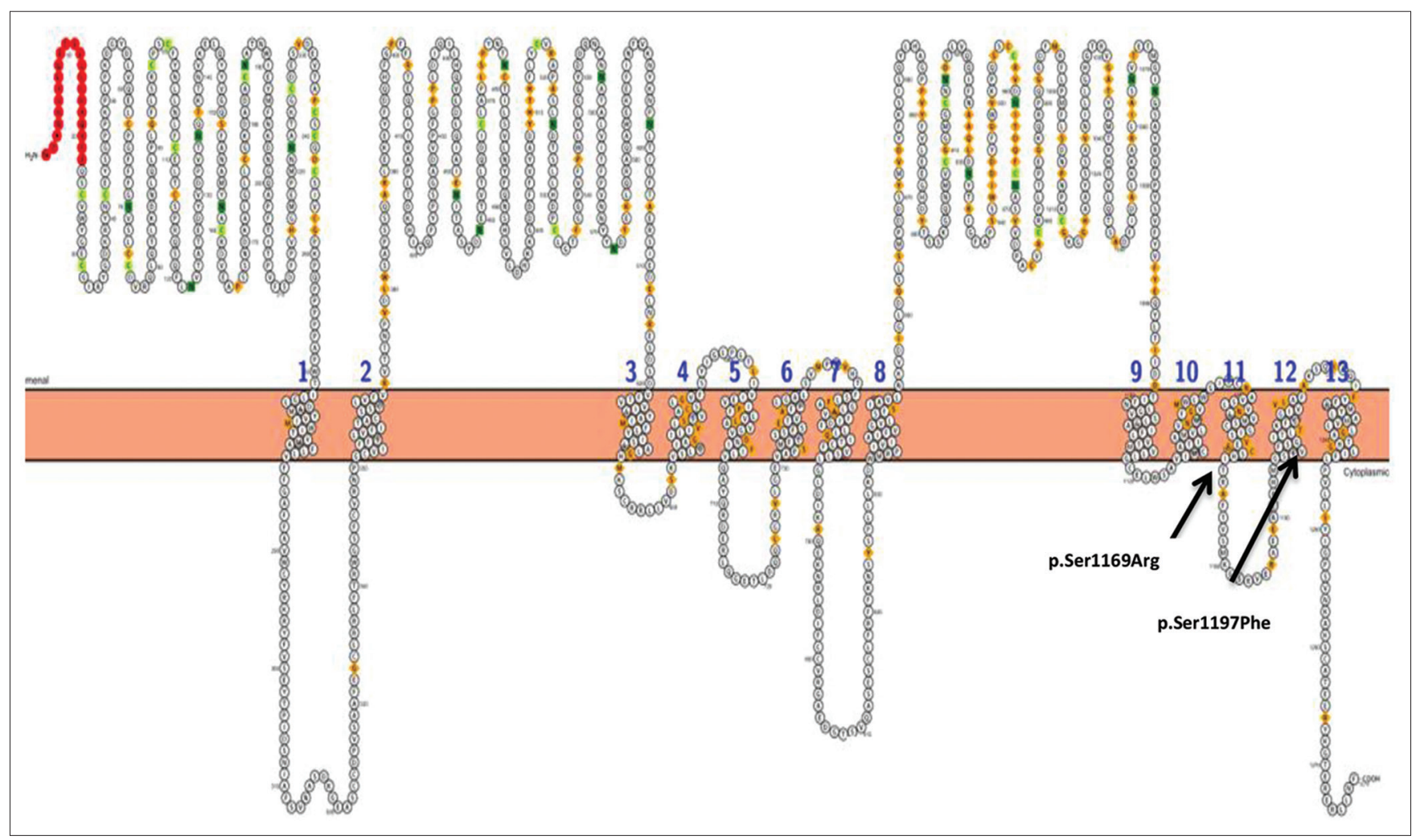

Figure S2: NPC1 protein topology and distribuition of the two novel missense variants (p.Ser1169Arg) and (p.Ser1197Phe). The 13 transmembrane domains of NPC1 are represented in the middle; cytosolic domains are shown at the bottom part, and luminal domains are shown at the upper part of the figure. Novel sequence alterations are indicated at their positions

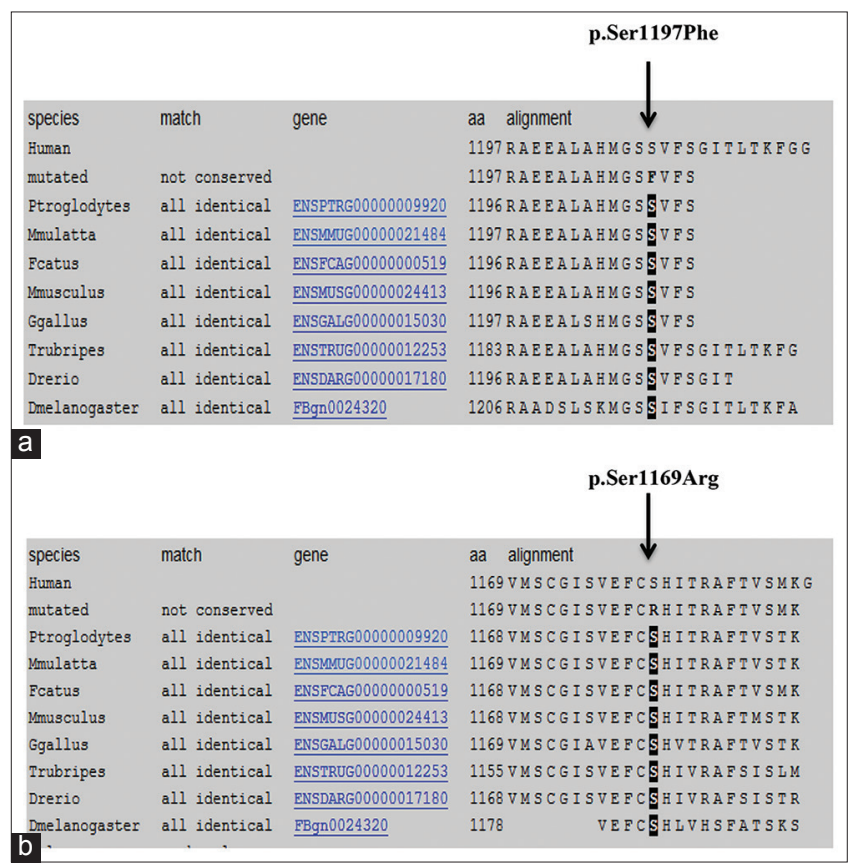

Figure S3: Cross-species conservation of the NPC1 protein sequence in relation to the two novel variants, (a) p.Ser1197Phe and (b) p.Ser1169Arg 
Table S1: Primer sequences used to amplify NPC1 exons

(17-25)

\begin{tabular}{|c|c|c|}
\hline Exon No. & Sequence & Fragment size \\
\hline $17 \mathrm{~F}$ & F: 5_-CCTGTACTCCCTATTAGCCTGTCAT-3_ & 322 \\
\hline $17 \mathrm{R}$ & R: 5_-ACTTGCTTGAAACACCTACGTGCATG-3 & \\
\hline $18 \mathrm{~F}$ & F:5_-TGCTTAGTTACTATCAGAGTGTTCAC-3_ & 291 \\
\hline $18 \mathrm{R}$ & R:5_-CCTCCTCCGCTGCTTCTGAAGTA-3_ & \\
\hline $19 \mathrm{~F}$ & F:5_-CTGTGGAGCAGGTCAGTAACCCT-3_- & 245 \\
\hline $19 R$ & R:5_-GTATAAACTGAGGCACGATGCAAATT-3_ & \\
\hline $20 \mathrm{~F}$ & F: 5_-CTTCTAACAGTCCTCCCTGCA-3_ & 247 \\
\hline $20 \mathrm{R}$ & R: 5_-CTGTCTTAGCCCAGTCCTCTC-3_- & \\
\hline $21 \mathrm{~F}$ & F: 5_-TGCTTAGCCTCAAGTGCTCAGAT-3_ & 337 \\
\hline $21 \mathrm{R}$ & R: 5_-ACCCAGTGTAGGCCCTTTGCTG-3_- & \\
\hline $22 \mathrm{~F}$ & F: 5_-GCGAGCTTTAATGAGGCCTCC-3_ & 295 \\
\hline $22 \mathrm{R}$ & R: 5_-GACAAACTGAGACTGTATGAGGA-3_ & \\
\hline $23 \mathrm{~F}$ & F: 5_-CAGGGTGCCCTGGGTAATTAGCA-3_- & 292 \\
\hline $23 R$ & R: 5_-GATCCAGACTCTTCAGTCACTGAG-3 & \\
\hline $24 \mathrm{~F}$ & F: 5_-TTCAATTACAGGTTGGTAAAAGTGGTT-3_ & 297 \\
\hline $24 \mathrm{R}$ & R: 5_-CTTGAAAAGAATGCCTCAGGATAGA-3_ & \\
\hline $25 \mathrm{~F}$ & F: 5_-TTCCAAAGTGGGATTACAGGCGTG-3_ & 221 \\
\hline $25 \mathrm{R}$ & R: 5_-GACCGACCCTTAGACACAGTTCAG-3_ & \\
\hline
\end{tabular}

Table S2: Biochemical analysis of 15 studied NPC patients

\begin{tabular}{|c|c|c|c|}
\hline Patient no. & $\begin{array}{l}\text { Chitotriosidase } \mu \mathrm{mol} / / / \mathrm{h} \\
\text { Reference level }(4-80 \mu \mathrm{mol} / \mathrm{L} / \mathrm{h})\end{array}$ & $\begin{array}{l}\text { Sphingomyelinase nmol/g.prot/h } \\
\text { Reference level (6-47 } \mu \mathrm{l} / \mathrm{g} . \mathrm{prot} / \mathrm{h} / \mathrm{g} . \mathrm{prot} / \mathrm{h})\end{array}$ & $\begin{array}{l}\beta \text {-glucocerebrosidase } \mu \mathrm{mol} / \mathrm{g} \text {.prot } / \mathrm{h} \\
\text { Reference level }(1-5 \mu \mathrm{mol} / \mathrm{g} / \mathrm{h})\end{array}$ \\
\hline 1 & 101.3 & 13.4 & 1 \\
\hline 2 & 88 & 23.4 & 1.5 \\
\hline 3 & 107 & 11 & 1.2 \\
\hline 4 & 282 & 30 & 4.7 \\
\hline 5 & 195 & 25 & 3.2 \\
\hline 6 & 171 & 7.3 & 2.6 \\
\hline 7 & 159 & 8.7 & 2.5 \\
\hline 8 & 242 & 7 & 3.5 \\
\hline 9 & 380 & 14 & 2.5 \\
\hline 10 & 195 & 25 & 3.2 \\
\hline 11 & 615 & 17 & 1.3 \\
\hline 12 & 329 & 7.3 & 2.8 \\
\hline 13 & 188 & 1.7 & 1.8 \\
\hline 14 & 215 & 13.7 & 5 \\
\hline 15 & 692 & 9 & 1.5 \\
\hline
\end{tabular}

